\title{
Dynamic Catalogue Enrichment with SeeAlso Link Servers
}

\author{
Jakob Voß \\ Verbundzentrale des GBV (VZG), Platz der Göttinger Sieben 1, \\ 37073 Göttingen, Germany. jakob.voss@gbv.de
}

\begin{abstract}
The poster presents architecture and usage of SeeAlso, a simple protocol for link servers that is used to dynamically enrich catalouges of libraries in the German Common library network GBV.
\end{abstract}

\section{Introduction and Related Works}

Links are the foundation of all hypermedia systems. In the WWW links are stored and managed in the source document they are pointing from. Centralized systems (for instance wikis) can manage links as objects of their own. Dedicated link servers can answer questions like what links point to a given resource (backlinks). Link servers on the Web are rare and limited to specific applications. The Open Hypermedia Protocol (OHP) for interoperability of hypermedia systems included the concept of link servers that provide links. 1] OpenURL is more used for resolving links. 2] Typed links on the Semantic Web are encoded with the Resource Description Framework (RDF) and SPARQL becoming its predominant query language. 3 More important to web applications are simple protocols like Pingback ${ }^{1}$ which is used to communicate the creation of a link. SeeAlso is such a simple protocol. It is used to query link servers for additional links and information that can then dynamically be integrated in library catalouges and other web pages.

\section{Specification and Implementation}

The main design goal of SeeAlso was ease of use and implementation. It mostly consists of the existing standards unAPI ${ }^{2}$ and OpenSearch Suggestions $3^{3}$ Both avoid the complexity of traditional library standards. OpenSearch Suggestions is an extension of OpenSearch to identify and describe search engines that return search term completions for a given search prefix (autocomplete). SeeAlso uses the same response format based on JavaScript Object Notation (JSON) to return a set of labels and links. Because a standard is useless without reliable and available implementations, a reference implementation of SeeAlso server and clients are provided as Open Source in Perl and JavaScript 4

\footnotetext{
1 http://hixie.ch/specs/pingback/pingback

2 http://unapi.info/

3 http://www.opensearch.org/

4 http://search.cpan.org/dist/SeeAlso-Server/
} 


\section{Usage and Implications}

Content delivered by a link server can either be integrated at the webserver or the browser dynamically processes it with JavaScript (AJAX). Both ways require a few lines of code. Lack of technical skills is still a large barrier to innovation in many libraries. That is why the SeeAlso client provides an additional method to include context links in any web page. The web application only has to link to the JavaScript client library and add a snippet of HTML such as:

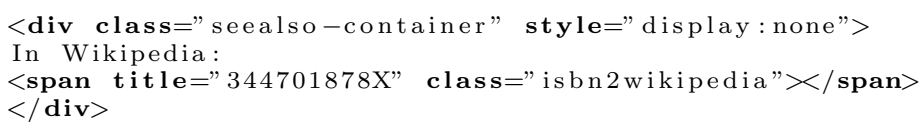

If the named link server (isbn2wikipedia) delivers links based on an identifier (344701878X), additional links to Wikipedia articles are shown. Identifiers are a basic feature of SeeAlso. Whenever possible, a normalized representation as URI should be defined, for instance for the ISBN 3-447-01878-X it is urn:isbn:9783447018784. The service center of the GBV library network provides a growing number of link servers:

- isbn2wikipedia: links to Wikipedia articles that cite a given book.

- pnd2gso: links to publications of a given author in the union catalog.

- hash2bibsonomy: links to BibSonomy for a given publication. A similar link server returns a tag cloud for the publication.

- isbn2librarything: link to LibraryThing (editions are joined).

- ppn2gbvbib: links to local library holdings for a given publication.

- isbn2gbs: link to a digitized version at Google Book Search.

Planned link servers will implement navigation in vocabularies and autocomplete via SeeAlso. Based on existing standards, the protocol is easy to implement and use. The loose coupling of link server and web application is part of a general strategy to focus on service oriented architecture (SOA) in digital libraries. With the SeeAlso link server protocol additional links to and between sources and services can be added with minimal effort. Thus several web 2.0 features can be integrated in our library catalouges without need to rebuild core parts of existing software.

\section{References}

1. Michaelides, D.T., Millard, D.E., Weal, M.J., Roure, D.D.: Auld Leaky: A Contextual Open Hypermedia Link Server. In: 12th ACM Conference on Hypertext and Hypermedia. Volume 2266 of LNCS., Springer (2001) 59-70

2. Van de Sompel, H., Beit-Arie, O.: Open Linking in the Scholarly Information Environment Using the OpenURL Framework. D-Lib Magazine 7 (2001)

3. Prud'hommeaux, E., Seaborne, A.: SPARQL Query Language for RDF. Technical report, W3C (2008) 